\title{
BMJ Open A qualitative focus group study of perceived barriers and benefits to exercise by self-described exercise status among older adults living with HIV
}

\author{
Nikolas A Johs, ${ }^{1}$ Yvonne Kellar-Guenther, ${ }^{2,3}$ Catherine M Jankowski, ${ }^{4}$ Hadlai Neff, ${ }^{3}$
} Kristine M Erlandson ${ }^{1}$

To cite: Johs NA, KellarGuenther Y, Jankowski CM, et al. A qualitative focus group study of perceived barriers and benefits to exercise by self-described exercise status among older adults living with HIV. BMJ Open 2019;9:e026294. doi:10.1136/ bmjopen-2018-026294

- Prepublication history for this paper is available online. To view these files, please visit the journal online (http://dx.doi org/10.1136/bmjopen-2018026294).

Received 24 August 2018 Revised 3 January 2019 Accepted 29 January 2019

Check for updates

(C) Author(s) (or their employer(s)) 2019. Re-use permitted under CC BY-NC. No commercial re-use. See rights and permissions. Published by BMJ.

${ }^{1}$ School of Medicine, University of Colorado, Aurora, Colorado, USA

${ }^{2} \mathrm{Cl}$ International, Center for Public Health Innovation, Littleton, Colorado, USA

${ }^{3}$ School of Public Health, University of Colorado, Aurora, Colorado, USA

${ }^{4}$ College of Nursing, University of Colorado, Aurora, Colorado, USA

Correspondence to Dr Kristine M Erlandson; Kristine.Erlandson@ucdenver. edu

\section{ABSTRACT}

Objectives Although exercise interventions have been shown to improve health outcomes among older people with HIV (PLWH), this population remains highly sedentary. The purpose of this study was to examine the differences in perceived barriers and benefits of exercise among older PLWH by self-identified exercise status.

Design Five focus groups were formed among PLWH: two groups of exercising men, two groups of non-exercising men and one group of women (mixed exercisers and nonexercisers). Themes were analysed in relation to the socialecological model, utilising the constant comparative approach. Setting Patients were recruited from an academic medical centre, HIV clinic and community locations. Participants PLWH aged 50 or older, diagnosed with HIV for at least 2 years, with no other health conditions that would preclude exercise.

Primary and secondary outcome measures Determine facilitators, barriers and the ideal environment for exercise or physical activity and determine whether these differ between older PLWH who self-identify as exercisers or non-exercisers.

Results Among 25 men (11 exercisers and 14 nonexercisers) and four women (three non-exercisers and one exerciser), non-exercisers mentioned fewer benefits of exercise $(n=46)$ than exercisers $(n=75)$. Exercisers emphasised positive reinforcement, positive mood change and increased energy as benefits of exercise; interpersonal benefits of exercise were also discussed twice as often by exercisers than by non-exercisers. Nonexercisers emphasised barriers to exercise including lack of motivation, lack of self-efficacy and a negative perception of gym culture. Non-exercisers identified the need for age-appropriate activities as a feature of an ideal exercise environment. Both groups identified time, cost and healthrelated challenges as barriers to exercise.

Conclusions Unique exercise barriers and benefits by self-identified exercise status provide important insights into the design of future interventions to initiate and maintain exercise.

Trial registration number NCT02404792; Results.

\section{INTRODUCTION}

With the advent of highly active antiretroviral therapy, nearly half of people living with HIV
Strengths and limitations of this study

- We explored thoughts about exercise adults with HIV and with and without established exercise habits, which has not previously been reported in the literature.

- This qualitative codebook was created in two phases. First, transcripts were read and coders created an initial coding scheme based on the patterns seen on the first pass-through. Additional codes were then developed from earlier focus groups in this population as well as themes from literature and added prior to analysis.

- All coding was done by hand by individual investigators, and all codes were reviewed by a third team member until a group consensus was reached.

- Results of the analysis were shared with members of the community advisory board for further validation of the data.

- Due to difficulty in recruiting women, data saturation of themes was not reached in this group and was only peripherally used to inform interpretation of the data.

(PLWH) are over 50 years old, and this number is expected to increase to $70 \%$ by $2030 .{ }^{1}$ Higher rates of age-associated comorbidities among older PLWH, such as cardiovascular disease, osteoporosis and neurocognitive disorders, ${ }^{2}$ are likely due in part to chronic inflammation seen with even suppressed HIV. ${ }^{3}$ Furthermore, older adults with HIV often face higher rates of depression, social isolation and the impact of both age-related and HIV-related stigma which contribute to health disparities. ${ }^{4-6}$ Exercise or physical activity appears to be a safe and effective way to prevent negative health outcomes associated with comorbidities in older PLWH. ${ }^{7-14}$ Exercise interventions have been shown to improve cardiovascular fitness, bone mineral density, body composition, functional status, neurocognitive function and health-related 
quality of life in PLWH, ${ }^{15-18}$ and exercise is recommended for older PLWH. ${ }^{19}$ Despite the importance of physical activity and exercise for health among older PLWH, high rates of sedentary behaviour are common. ${ }^{20} 21$

The factors that drive health behaviours in a population are often described through the social-ecological model (SEM). The SEM posits that health behaviour is influenced by a number of personal, social, organisational, community and policy-level factors that interact, and cannot be fully understood or enacted on without addressing all potential levels of influence..$^{22}{ }^{23}$ Indeed, the SEM model has been used extensively in the exercise health- behaviour literature to investigate the many competing factors that influence physical activity. ${ }^{24-27}$ Similarly, a number of qualitative studies have explored attitudes towards exercise or physical activity among diverse populations of PLWH and have identified factors influencing exercise or routine physical activity, such as physical health and self-efficacy (personal), social support and competing priorities such as caregiver responsibilities (social), and accessibility of safe environments for exercise (community) ${ }^{28-33}$ However, these studies did not distinguish the viewpoints of participants with and without established exercise habits. Thus, the focus of this qualitative study was to explore whether the facilitators, barriers and the ideal environment for exercise or physical activity differ between older PLWH who self-identify as exercisers and non-exercisers, with the hypothesis that many factors directly influencing exercise behaviour would span the SEM levels. Identification of the key differences influencing behaviour in PLWH who do and do not exercise will inform multi-level interventions to most effectively transition non-exercisers to self-identified exercisers.

\section{METHODS}

Participants were recruited from a university-based HIV primary care clinic and from local HIV community groups. Eligible participants were diagnosed with HIV for $\geq 2$ years, aged $\geq 50$ years, regularly seeing a physician for HIV care and taking antiretroviral therapy for $\geq 6$ months. Exclusion criteria included inability to provide consent, inability to speak English and inability to participate in any form of exercise. Participants self-identified exercise behaviour in response to the question, "Are you currently exercising regularly, meaning more than 2 days per week, on most weeks?".

Five focus groups were conducted in June 2016 including two groups of men that self-identified as exercisers, two groups of men that self-identified as non-exercisers and a group of women (both exercisers and non-exercisers, $n=4)$. The focus groups took place in a private conference room in an outpatient medical facility located near the clinic where the majority of participants obtained care. All five focus groups were conducted by a physician who worked in the HIV primary care clinic (KME); four of the participants were patients of the physician conducting the focus group. Notes were taken by a second investigator with no treatment relationship to the participants (NAJ). Participants completed a short demographic survey prior to the focus group session. Each session lasted approximately 2 hours. A semi-structured guide of open-ended questions informed by literature and prior work by our group ${ }^{34}$ was used to facilitate the discussion. All focus groups were audio-recorded and transcribed verbatim by a transcriptionist not involved in the study. Participants were provided with a $\$ 20$ gift card after completion of the focus group.

The transcripts were analysed individually by two members of the study team involved in the focus groups and another team member who was not involved. First, rather than starting with a theory like SEM, the team created a codebook in two phases. First, transcripts were read and coders created an initial coding scheme based on the patterns seen on first pass-through. Codes were then added using a deductive approach with a list of key domains developed from a prior set of focus groups (in PLWH), as well as prior literature for exercise barriers in older adults and PLWH. ${ }^{34}$ New codes identified during the analysis were added to the coding scheme and, utilising a constant-comparison approach, the early transcripts were reanalysed after the final codebook was determined.

Coding and analyses were completed by hand, independently by each investigator, and then codes were compared; differences were resolved by group consensus. Categories were structured by benefits and motivators of exercise, barriers to exercise, ideal conditions for exercise and other factors. These categories were then analysed and compared between the self-identified exercisers and non-exercisers. In the results, a note was made when a code was mentioned by all four focus groups or by both focus groups of each type (exercisers or non-exercisers). Codes that were unique to only one type (exercisers or non-exercisers) were then compared with literature to determine if saturation was reached. Challenges with recruitment of women to the study did not allow for their subdivision into self-identified exercisers and non-exercisers and because data saturation could not be reached, themes from this group were used to support findings of the other groups. Quotes from the interviews are provided for insight into the identified themes.

\section{Patient and public involvement}

Member-checking involved review and discussion of the results with seven men who are part of our community advisory group. These men reflect characteristics of the focus group participants and provided input as to whether the focus group results resonated with their experiences.

\section{RESULTS}

Twenty-nine participants (table 1) from five focus groups included 11 men who self-identified as exercisers (focus groups $\mathrm{n}=6, \mathrm{n}=5), 14$ men who self-identified as non-exercisers (focus groups $n=8, n=6$ ), and four women. Briefly, among the men, the median age was 57 years, the majority 
Table 1 Participant characteristics

\begin{tabular}{|c|c|c|c|}
\hline & $\begin{array}{l}\text { Exercising } \\
\text { Men }(n=11)\end{array}$ & $\begin{array}{l}\text { Non- } \\
\text { exercising } \\
\text { Men }(n=14)\end{array}$ & $\begin{array}{l}\text { Women (Non- } \\
\text { exercisers and } \\
\text { exercisers, } n=4 \text { ) }\end{array}$ \\
\hline Age $^{*}$ & $57(53,61)$ & $58(53,65)$ & $56(53,61)$ \\
\hline \multicolumn{4}{|l|}{ Race† } \\
\hline White & $10(91)$ & $8(57)$ & $2(50)$ \\
\hline Black & 1 (9) & $3(21)$ & $1(25)$ \\
\hline Other & $0(0)$ & $3(21)$ & $1(25)$ \\
\hline \multicolumn{4}{|l|}{ Ethnicity† } \\
\hline Hispanic or Latino & 1 (9) & $2(14)$ & $1(25)$ \\
\hline Not Hispanic/Unknown & $10(91)$ & $12(86)$ & $3(75)$ \\
\hline \multicolumn{4}{|l|}{ Educationt } \\
\hline Some high school & $0(0)$ & $0(0)$ & $1(25)$ \\
\hline High school or equivalent & $4(36)$ & $2(14)$ & $0(0)$ \\
\hline Some college & $3(27)$ & $5(36)$ & $3(75)$ \\
\hline College degree & $3(27)$ & $2(14)$ & $0(0)$ \\
\hline Post-graduate work & 1 (9) & $5(36)$ & $0(0)$ \\
\hline \multicolumn{4}{|l|}{ Employment† } \\
\hline On disability & $6(55)$ & $4(29)$ & $0(0)$ \\
\hline Unemployed & $0(0)$ & $5(36)$ & $3(75)$ \\
\hline Retired & $3(27)$ & $4(29)$ & $0(0)$ \\
\hline Full-time & $2(18)$ & $2(14)$ & $0(0)$ \\
\hline Part-time & $0(0)$ & $0(0)$ & $1(25)$ \\
\hline $\begin{array}{l}\text { Years since HIV } \\
\text { diagnosis* }\end{array}$ & $20(14,27)$ & $20(11,27)$ & $15(4,27)$ \\
\hline \multicolumn{4}{|l|}{ Sexual preference $\dagger$} \\
\hline Men & $8(73)$ & $13(93)$ & $4(100)$ \\
\hline Women & $2(18)$ & $1(7)$ & $0(0)$ \\
\hline Other & 1 (9) & $0(0)$ & $0(0)$ \\
\hline \multicolumn{4}{|l|}{ Self-reported comorbidities $† \ddagger$} \\
\hline Hypertension & $4(36)$ & $4(29)$ & $2(50)$ \\
\hline Hyperlipidaemia & $4(29)$ & $2(14)$ & $0(0)$ \\
\hline Diabetes & $0(0)$ & $2(14)$ & $0(0)$ \\
\hline Depression/anxiety† & 7 (64) & $9(64)$ & $3(75)$ \\
\hline Osteoporosis $\dagger$ & $0(0)$ & $1(7)$ & $0(0)$ \\
\hline
\end{tabular}

Presented as *median with IQR or tfrequency (\%).

łLanguage used in the survey instrument for self-reported comorbidities was as follows: Have you ever been told by a doctor that you have any of the following medical problems? High blood pressure, high cholesterol,

diabetes, depression or anxiety, osteoporosis.

were white and college-educated men who have sex with men. The women had a median age of 56 years, the majority having some college education and sexual preference for men. Male participants had been living with HIV for approximately 20 years (range of 4 to 33 years), and female participants for 15 years (range 4 to 27 years).

\section{Motivators of and benefits of exercise}

A variety of motivators of exercise and benefits of exercise were reported by both exercising and non-exercising groups although, overall, non-exercisers mentioned far fewer benefits of exercise $(n=46$ mentions) than exercisers $(n=75$ mentions), table 2. A shared theme was the benefit of being able to think more clearly, or a sense of self-reflection or meditation. For the exercisers, this was more often related to feeling mindful during exercise: "swimming is something like putting yourself in a meditative state. Which is relaxing and you know...it gives me a chance just to zone out by myself." The non-exercising group spoke about how physical activity helped them think more clearly: "...there is a physiological and mental aspect to it that is very refreshing. It [exercising] is good for your soul... It clears your mind." Exercisers perceived a benefit in mood, mental health and coping with stress. As one participant mentioned, "I was diagnosed bipolar about 18 years ago. And so I use exercise as a coping mechanism for when I'm depressed or when I'm manic...It grounds me." Exercise also helped combat the effects of ageing: "Well, I'm 60 and I didn't want anything headed south on me!"

The social aspects of exercise were recognised more commonly than personal benefits in all groups, and nearly twice as often among exercisers: "...having a friend or a buddy to exercise with is a great motivator," (exerciser). Exercisers placed more importance on the motivation and encouragement or positive reinforcement from family or peers: "Especially like people at church who I see every Sunday and they are like, keep doing it. Keep going. Keep going. You look great. But that positive reinforcement you know, building my self-esteem a little bit more and you know, remembering that makes me want to walk that half mile ...Cause then I know it's paying off," (exerciser). Non-exercisers reported the influence of the social environment or the community on motivation to start exercise. For example, one participant mentioned how much more motivated he was to be active when his community was more active, "not everybody can live in the mountain towns [more physically active towns] and they don't want to. But anyway I had a great network of friends up there. Socially and sports-wise," (non-exerciser). Additional motivators and benefits are detailed in table 2.

\section{Barriers to exercise}

Prominent barriers discussed almost exclusively by the non-exercising group included lack of motivation or discipline, lack of a daily routine and difficulty related to fatigue or other life-struggles: "Sometimes it takes everything I have to get out of bed in the morning." Poor exercise self-efficacy was also more commonly discussed among non-exercisers than exercisers. One participant described a strong desire to be active for health reasons but faced challenges in establishing a regular workout routine, "But the motivation to believe in myself and to have the ability to know that I can do it again, would probably be the hardest part for me." This also coincided with a lack of familiarity about exercise or lack of knowledge of newer technology, "First of all, there are technological advances that have happened since I spent any time in a gym. And I think...that when it comes to being inside a gym or something, I don't know what the best order of machines would be. Or what is going to work for you." Age-related barriers further contributed to low exercise self-efficacy. When one participant asked the group if 


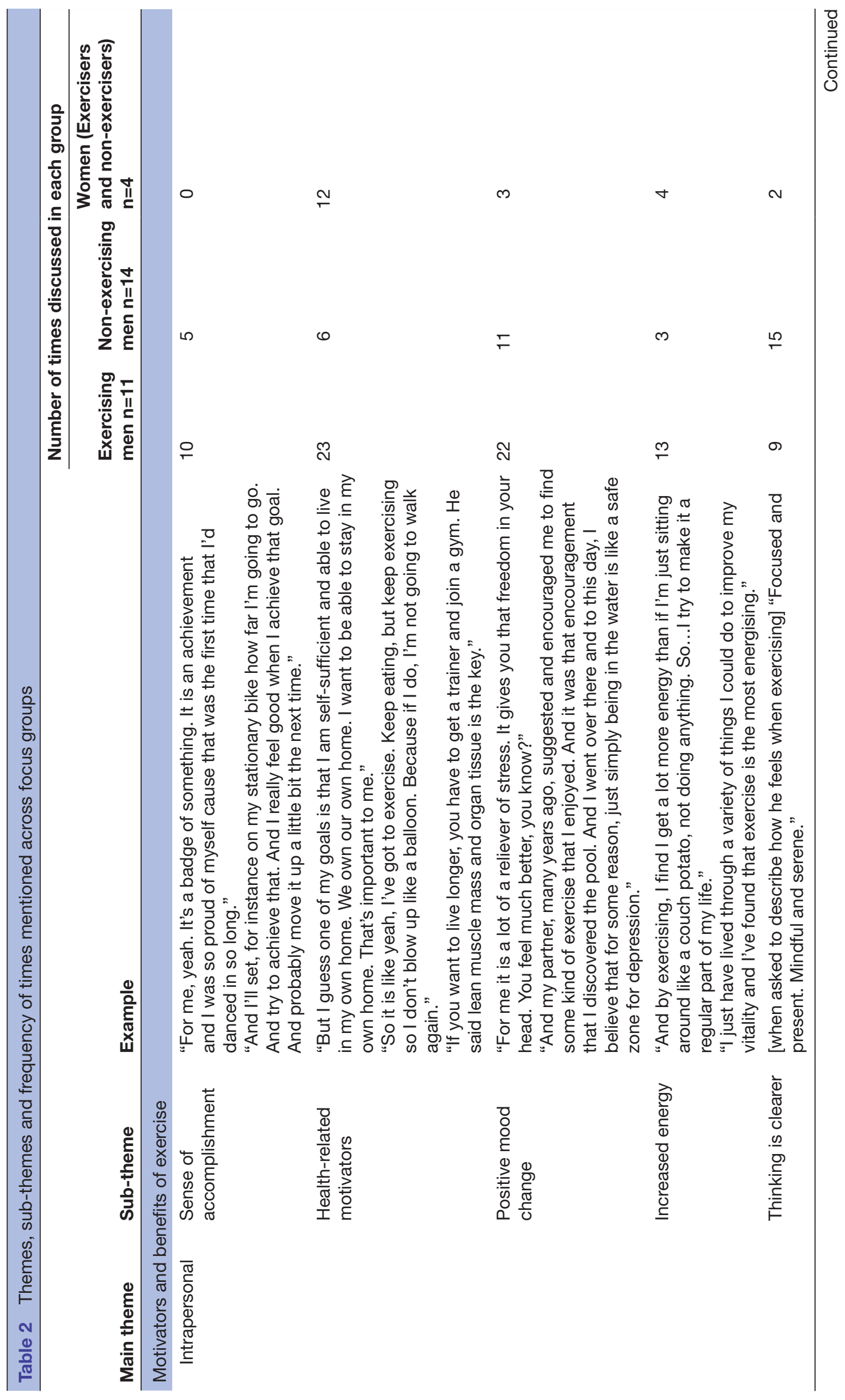




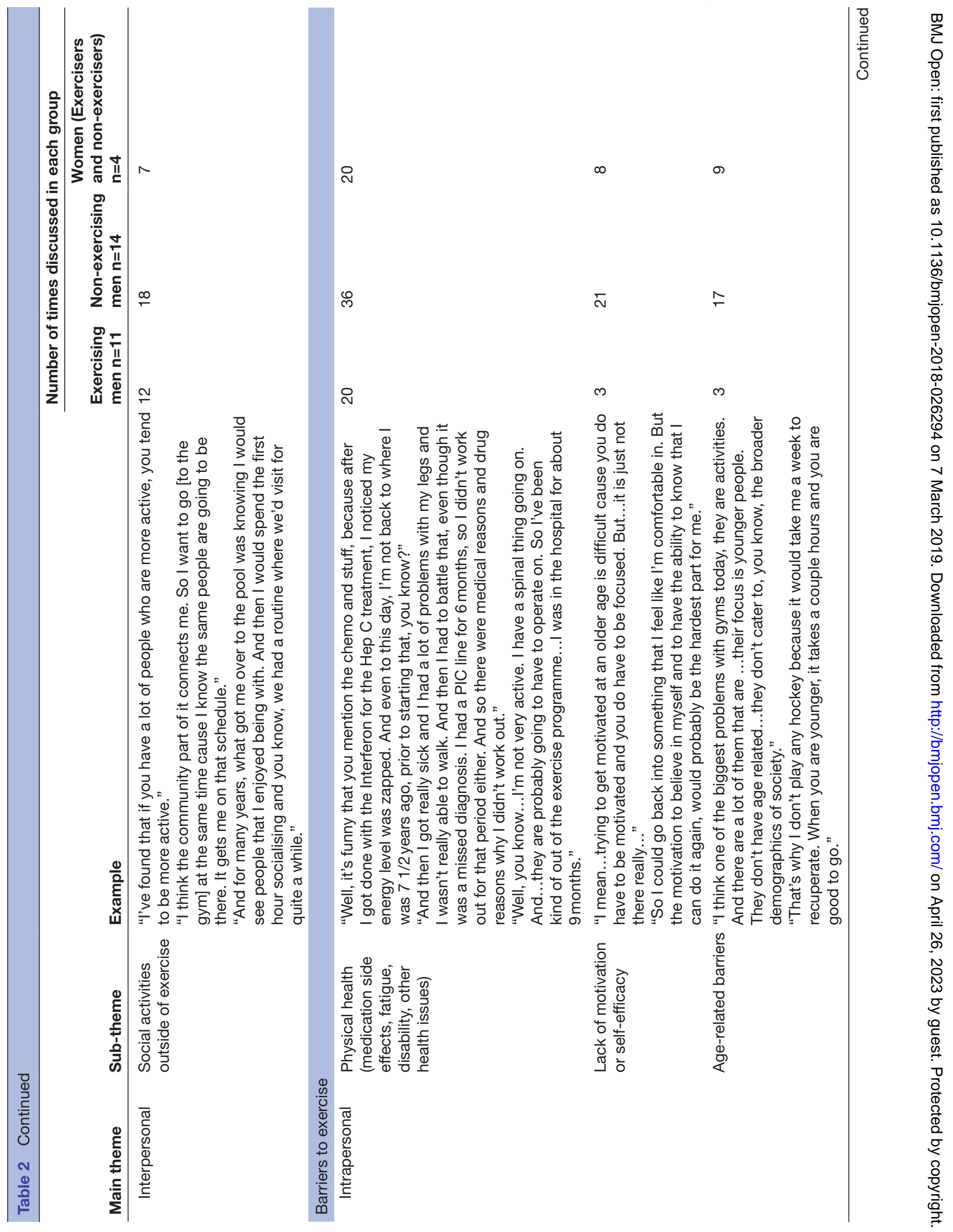




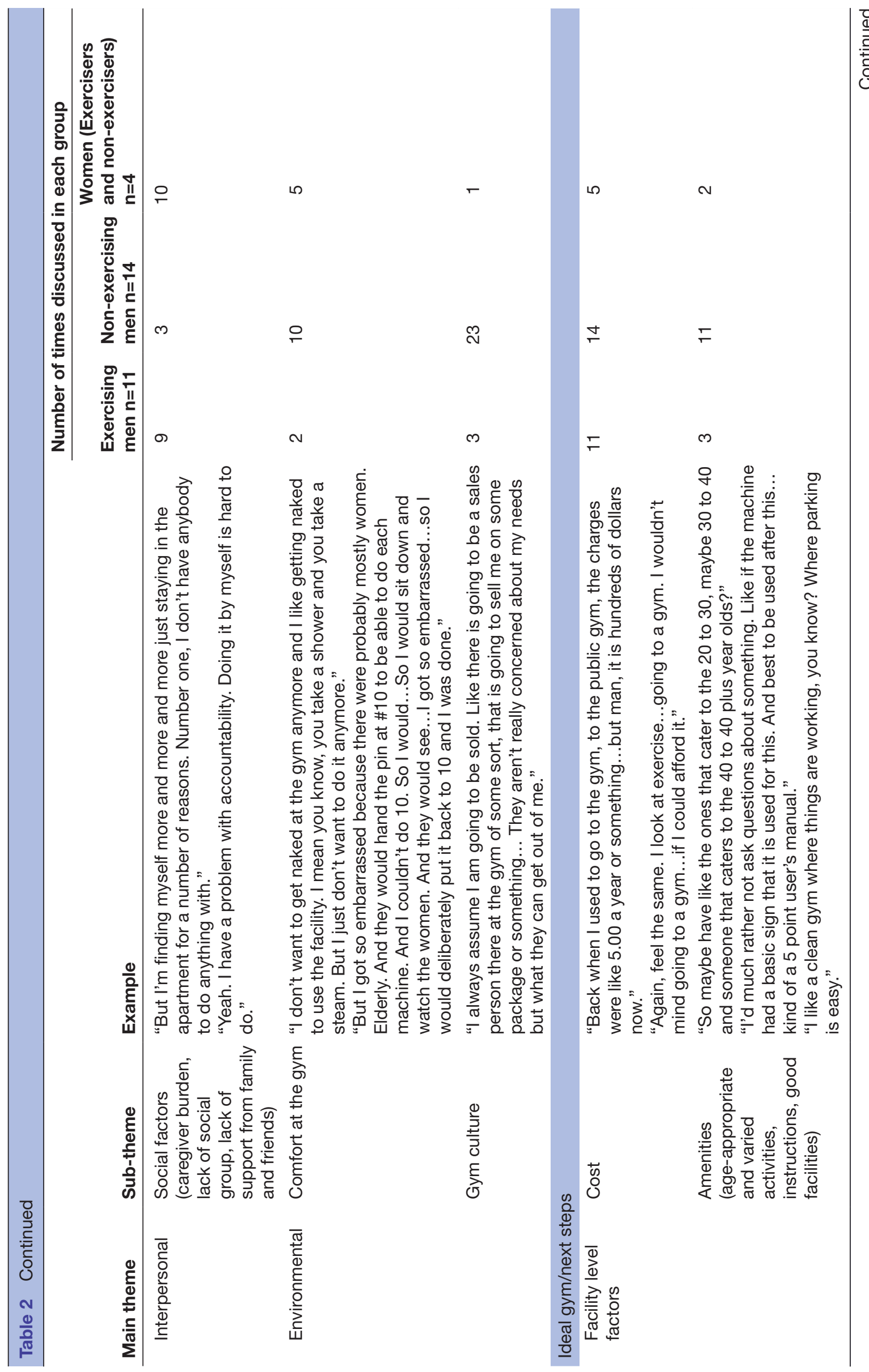

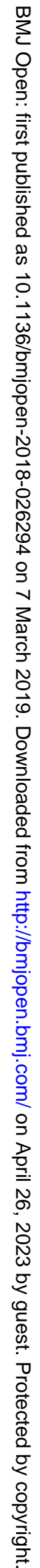




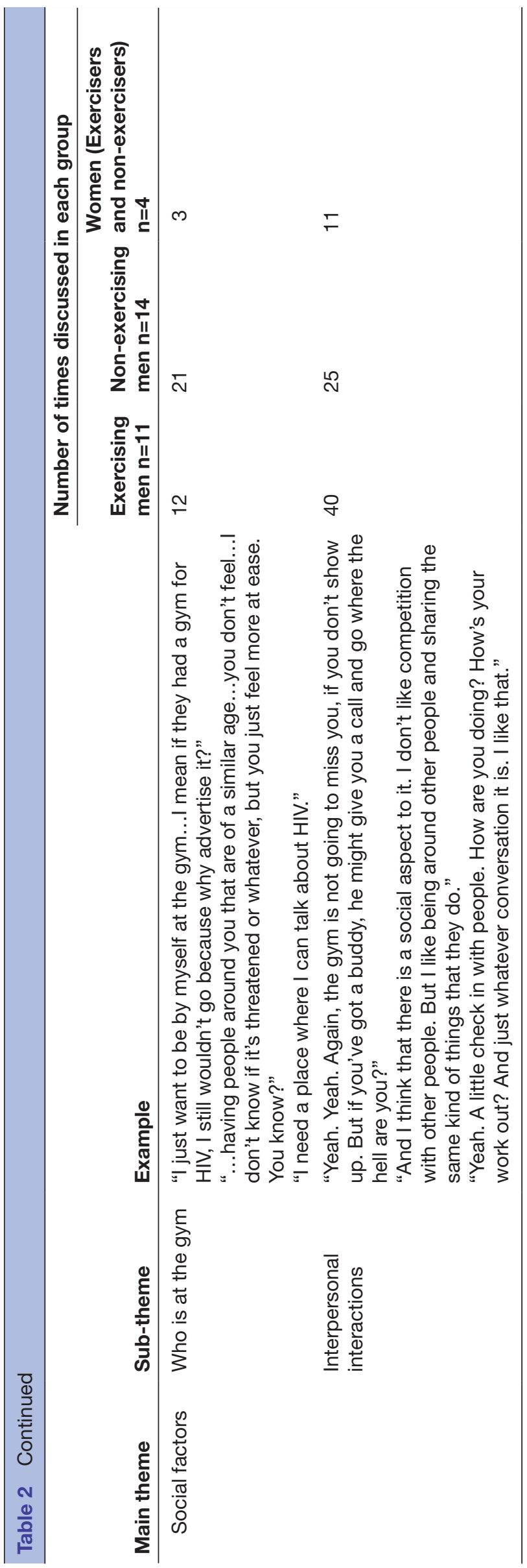

he was the only one that felt fatigue, another replied, "I don't have a bundle of energy. I'm not bouncing off the walls, you know? Twenty years ago, I used to bounce off the walls "cause I had so much [energy]..." One group particularly bemoaned the lack of age-appropriate activities at gyms: “...they are not going to be able to fine-tune something that is more appropriate for someone my age or with various health issues and things like that."

Physical health, fatigue, injuries and hospitalisation were mentioned among both groups, but more commonly identified as barriers among non-exercisers. One patient related the physical health barriers following a hospitalisation: "I ended up being in the hospital for a week with septic shock...up until that time, I was $100 \%$ fine...and then the next thing I know, I'm in the intensive care unit and obviously after a week in the hospital, I was much weaker. But when I came home it was like wait, why am I getting winded? So my mind said I should be able to continue doing things at the level I was before. But my body wasn't doing that," (non-exerciser). Other themes discussed by both groups included having a lack of time ("work is just really an impediment," non-exerciser), depression and loss of a personal relationship ("when he left me, it zapped everything," (non-exerciser).

Uniquely identified in the non-exercising groups was a negative perception of gym culture. Participants expressed disdain for younger gym members who were perceived to be more focused on their looks than health ('posers' was the term used by one participant) and for the general environment: "Another thing that really turned me off was during a couple decades period of time, the gym culture changed dramatically from just being a work-out place, to being these entertainment centres with pounding music and all this fancy gym clothes... one day I just walked in and the music was pounding and I said, you know, you got all these frickin' queens posing like peacocks in the mirrors and I've had it." The gym was also perceived as a business more focused on selling a product than health, "I don't think they are interested in my health as much as they are trying to sell themselves and make money. I don't like that at all. Because they are not interested in me." In contrast to these negative perceptions expressed by non-exercisers, exercisers commented more on the aspects of community within the gym that they enjoyed.

Exercisers noted lack of social support as a barrier to exercise. When talking about the time period prior to being an exerciser, a participant related, "I had never lived for me. It was always about my partner or my parents or my teachers, or my friends. My whole life - 50 years - had revolved around what somebody else wanted for me." Another expression of this theme was related to simply not having a social group with which to participate in an activity, "...Before my partner passed away, we used to like to go up in the mountains and go hiking. But right now it is like...I don't know anybody around here that enjoys doing it. So this summer I haven't gone hiking at all. I take [my dog] out for his walks. But it's like...I just, you know...I don't know anybody to go hiking with so..." 


\section{Ideal environment for exercise}

When participants were asked to describe ideal exercise conditions, many of the answers related back to motivators and barriers (table 2). For both groups, cost and access were important factors. Multiple groups mentioned benefits of insurance-sponsored programmes and reducedcost community centres, and expressed intentions of investigating such programmes. The type of exercise was also related to cost for some participants (exerciser) : "I actually kind of enjoy going out for a run. It doesn't cost me anything. It's free." Interpersonal and social factors were important factors for both groups, with every group describing an aspect of the social environment that it thought was important, whether an 'exercise buddy' or a welcoming environment: "If someone was genuinely interested in working with me and when I say, generally interested in it from a social perspective. You know?" (non-exerciser).

We also posed the question of what the preferred exercise community would be. Opinions differed across groups: some felt that they would appreciate having a gym that catered to PLWH exclusively whereas others were opposed to this idea. Non-exercisers preferred a gym or programme that catered to older adults: “... having people around you that are of a similar age. You know? You don't feel ...I don't know if it's threatened or whatever, but you just feel more at ease. You know?" Non-exercisers were also more interested in the types of activities available at the gym and the facilities, and in particular, mentioned the importance of having age-appropriate activities. When describing an acceptable gym format, one participant stated, "what I liked about [the gym]... all the machines are in a circle and it's just for seniors." This group also mentioned dedicated instructions as something that would be a part of their ideal gym; or clear instructions that would help them get started if they were not familiar with a given exercise.

\section{Other factors acting as barriers to exercise or physical activity}

Throughout the discussion of physical activity and exercise, many other factors were acknowledged, though not directly in the context of exercise or physical activity. These topics related to life's challenges that the participants faced, including drug abuse, feeling unsafe in their neighbourhood, lack of affordable housing, mental health issues, or stigma related to HIV, sexual preference or age. Every group had unprompted discussion about social isolation, or loss of friends or partners due to HIV or other reasons: "Now that I'm older, I don't have any 'gay friends' anymore. And I wonder, is it me? Or have they gone somewhere? I mean a lot of them died...But like, I don't have any gay friends. My friends are accepting of me even they know I am, but I don't have any. Do you all have any friends who are HIV positive?" This limited group of friends threatens an important social support motivator for exercise.

\section{DISCUSSION}

As SEM is frequently used to inform the personal and societal factors that influence physical activity, we anticipated that our participants would identify the contribution of each level to their own exercise and physical activity behaviours. ${ }^{222526}$ Instead we found that participants focused much more on the personal, physical, mental and social factors. While environment came up as a deterrent for non-exercisers (negative gym culture), policy was seldom recognised as an influence on physical activity. The most salient theme identified that may serve as a target for future policy-level changes was access (cost and transportation) to a gym, which was identified as a barrier by both exercisers and non-exercisers. The lack of connection with the gay community was also mentioned, but participants did not directly connect that to physical activity. Furthermore, exercisers and non-exercisers recognised some similar, but also unique, barriers and facilitators to routine exercise or physical activity that are important for consideration when designing lifestyle interventions for older PLWH.

Physical health was identified as both a major barrier and a motivating factor for exercise. Exercisers were more likely to mention the health-related benefits of exercise, report increased energy and acknowledge the importance of exercise towards improving overall health, as has been reported previously in the general exercise literature. ${ }^{26}$ Despite the medical advances in HIV management with antiretroviral therapy, older PLWH appear to experience age-associated comorbidities at a higher rate and at a younger age than the general population. ${ }^{235}$ The physical barriers attributed by our participants to HIV and medication, generalised fatigue, and chronic pain are consistently identified as barriers to exercise among PLWH. ${ }^{28} 31-33$ T6 To engage older PLWH, exercise interventions must acknowledge and address these physical barriers.

All groups discussed the impact of depression and loss in their exercise behaviours (or life) but the positive impact of exercise on mood and mental health was most often recognised by the exercisers. These results are consistent with a recent meta-analysis that found that aerobic and resistance exercise interventions had positive effects on various domains of mental health such as stress, anxiety and depressive symptoms in PLWH. ${ }^{37}$ In contrast, mental health related barriers to physical activity are well-described among other populations of PLWH, ${ }^{28} 3233$ and depression has been correlated with decreased PA levels in both PLWH and in the general population. ${ }^{2638}$ Depression as a barrier to exercise is of particular relevance given that PLWH have up to three times the rate of depression as the general population, ${ }^{5}$ and that perceived well-being may be associated with adherence to an exercise regimen. ${ }^{39}$ Among exercisers, the mental health benefits of exercise were an important factor in the maintenance of physical activity and, thus, an important domain to emphasise in future exercise intervention studies.

One of the more striking differences between exercisers and non-exercisers was related to the lack of exercise motivation and exercise self-efficacy among 
non-exercisers. Self-efficacy has consistently been identified as one of strongest correlates of exercise behaviour in adults in general ${ }^{25-27} 4041$ and in PLWH. ${ }^{38}$ Self-efficacy is commonly defined as an individual's confidence in his/ her ability to complete a task or series of actions, in this case the ability to exercise safely and effectively. Self-efficacy may interact with motivation for exercise, such that an individual with poor exercise self-efficacy has poor recognition of exercise benefits, less confidence in exercise ability, and therefore, less motivation to initiate exercise. ${ }^{42}$ The non-exercising group identified a number of themes related to poor self-efficacy, including changing technology in gyms, older age and poor health. Similarly, non-exercisers identified interventions that may help overcome the lack of self-efficacy, including access to specific instructions or wellness facilities that cater to older adults.

The social aspects of exercise or physical activity were strong motivators or benefits for both groups. Exercisers were more likely to mention encouragement from others as an important motivator, whereas non-exercisers focused on the general social environment rather than the exercise-specific environment. Social isolation and a desire to connect with other social groups were discussed by all participants, though not directly in the context of exercise. The importance of social support for exercise among PLWH has been described, whether as a 'workout buddy', general community activities or more social support in general. ${ }^{2893233}$ Older PLWH have high rates of social isolation and may have challenges related to loss of friends due to illness or estrangement from family due to HIV or other stigma. ${ }^{6}{ }^{43}$ Social support has been associated with greater physical activity and loneliness with decreased physical activity. ${ }^{44}$ Effective physical activity initiation and maintenance among older PLWH may be strengthened by a robust social environment.

A unique finding was the negative perception of gym culture, recognised to the greatest extent among non-exercisers. The younger community at the gym was perceived as non-welcoming, and participants desired a genuine community that connected with them as individuals rather than as depersonalised customers. There was little consensus among our focus groups regarding what an ideal exercise or a physically active community would look like, particularly among the non-exercising group. In our previous work among older men with HIV enrolled in an exercise intervention, participants discussed the appeal of a gym community for older PLWH. ${ }^{34}$ Here, however, we found this concept to be contentious, some citing a desire for connection with similar others (older and/or with HIV), but others relating a desire for privacy and expressing concerns about disclosure due to HIV stigma, noted as a barrier to exercise in prior studies of PLWH. ${ }^{29}{ }^{31}$ Based on the data from these focus groups, the welcoming environment of a new gym or activity may be particularly important to those not currently exercising, and activities targeted for older adults may be beneficial. Aside from gym culture, the role of organisational, community, and policy factors (access, cost, indirect barriers such as insecure housing and affordable food, and the built environment) were discussed but not dominant. These commonly reported barriers to physical activity in the general population are the focus of public health and policy interventions. ${ }^{2426274546}$

Our study has several limitations. The majority of our participants were white college-educated men, limiting the generalisability of our findings. Themes mentioned by the women's group that merit further exploration include the importance of health-related barriers to exercise and placing a higher value on an active lifestyle as opposed to structured exercise. Additionally, our participants were self-identified as exercisers or non-exercisers with no objective verification of the actual amount or type of their physical activity. While many of the themes our participants discussed are supported by the literature, ideas about barriers due to gym culture and the business models have not been reported to our knowledge, and our focus groups may not have been sufficient to provide saturation for these specific topics. When these results were reported to our community advisory group, they agreed that many corporate gyms had an unfriendly environment, and that, perhaps, smaller recreation centres might have the more welcoming atmosphere that our participants desired.

\section{CONCLUSIONS}

In summary, interpersonal and intrapersonal factors, including an emphasis on social connections, were central to exercise behaviours among older adults with HIV whereas organisational, community and policy level factors of the SEM were minor contributors. While addressing these higher-level SEM domains may be important for long-term and large-scale exercise and physical activity dissemination and maintenance, strategies to promote physical activity in older PLWH may need to focus on overcoming physical barriers and providing a safe and welcoming social environment.

\section{Acknowledgements Sarah Dunham, MPH also coded data.}

Contributors NAJ, HN, and KME developed the initial codebook, with input from CMJ. KME led the interview sessions with additional input from NAJ and CMJ. Data coding was completing by Sara Dunham (see above), NAJ, and KME, with input from CMJ, YK-G, and HN if consensus was not achieved. NAJ prepared the first manuscript draft; all authors contributed to, reviewed, and approved the final manuscript.

Funding This research was funded through the National Institutes of Health, National Institute on Aging (K23 AG050260 to KME) and NCATS Colorado CTSA (UL1 TR002535).

Disclaimer Contents are the authors' sole responsibility and do not necessarily represent official NIH views.

Competing interests KME has received funding for research paid to the University of Colorado from Gilead Sciences, and has received funding for advisory panel from Viiv Healthcare.

Patient consent for publication Not required.

Ethics approval The study was approved by the Colorado Multi-Institutional Review Board.

Provenance and peer review Not commissioned; externally peer reviewed.

Data sharing statement Although the transcripts have been de-identified, to protect participant confidentiality regarding some conversations that occurred 
during the focus groups, the transcripts are not readily available. Codebooks or sections of the transcripts can be requested by contacting the corresponding author.

Open access This is an open access article distributed in accordance with the Creative Commons Attribution Non Commercial (CC BY-NC 4.0) license, which permits others to distribute, remix, adapt, build upon this work non-commercially, and license their derivative works on different terms, provided the original work is properly cited, appropriate credit is given, any changes made indicated, and the use is non-commercial. See: http://creativecommons.org/licenses/by-nc/4.0/.

\section{REFERENCES}

1. Smit M, Brinkman K, Geerlings S, et al. Future challenges for clinical care of an ageing population infected with HIV: a modelling study. Lancet Infect Dis 2015;15:810-8.

2. Deeks SG. HIV infection, inflammation, immunosenescence, and aging. Annu Rev Med 2011;62:141-55.

3. Castillo-Mancilla JR, Brown TT, Erlandson KM, et al. Suboptimal Adherence to Combination Antiretroviral Therapy Is Associated With Higher Levels of Inflammation Despite HIV Suppression. Clin Infect Dis 2016;63:1661-7.

4. Kaufman MR, Cornish F, Zimmerman RS, et al. Health behavior change models for HIV prevention and AIDS care: practical recommendations for a multi-level approach. J Acquir Immune Defic Syndr 2014;66(Suppl 3):S250-8.

5. Do AN, Rosenberg ES, Sullivan PS, et al. Excess burden of depression among HIV-infected persons receiving medical care in the United States: data from the medical monitoring project and the behavioral risk factor surveillance system. PLoS One 2014;9:e92842.

6. Emlet CA. An examination of the social networks and social isolation in older and younger adults living with HIV/AIDS. Health Soc Work 2006;31:299-308.

7. Botros D, Somarriba G, Neri D, et al. Interventions to address chronic disease and HIV: strategies to promote exercise and nutrition among HIV-infected individuals. Curr HIVIAIDS Rep 2012;9:351-63.

8. O'Brien KK, Tynan A-M, Nixon SA, et al. Effectiveness of aerobic exercise for adults living with HIV: systematic review and metaanalysis using the Cochrane Collaboration protocol. BMC Infect Dis 2016;16:182.

9. O'Brien KK, Tynan AM, Nixon SA, et al. Effectiveness of Progressive Resistive Exercise (PRE) in the context of HIV: systematic review and meta-analysis using the Cochrane Collaboration protocol. BMC Infect Dis 2017;17:268.

10. Nixon $\mathrm{S}, \mathrm{O}^{\prime}$ Brien $\mathrm{K}$, Glazier $\mathrm{RH}$, et al. Aerobic exercise interventions for people with HIV/AIDS. Cochrane Database Syst Rev 2001;1:Cd001796.

11. Nixon S, O'Brien K, Glazier $\mathrm{RH}$, et al. Aerobic exercise interventions for adults living with HIV/AIDS. Cochrane Database Syst Rev 2002;2:Cd001796.

12. O'Brien K, Nixon S, Glazier R, et al. Progressive resistive exercise interventions for adults living with HIV/AIDS. Cochrane Database Syst Rev 2004;15:Cd004248.

13. Nixon $\mathrm{S}$, O'Brien $\mathrm{K}$, Glazier $\mathrm{RH}$, et al. Aerobic exercise interventions for adults living with HIV/AIDS. Cochrane Database Syst Rev 2005;2:Cd001796.

14. O'Brien K, Nixon S, Tynan A-M, et al. Aerobic exercise interventions for adults living with HIV/AIDS. Cochrane Database Syst Rev 2010;13:Cd001796.

15. Jaggers JR, Hand GA, Dudgeon WD, et al. Aerobic and resistance training improves mood state among adults living with HIV. Int $J$ Sports Med 2015;36:175-81.

16. Gomes Neto M, Conceição CS, Oliveira Carvalho V, et al. Effects of combined aerobic and resistance exercise on exercise capacity, muscle strength and quality of life in HIV-infected patients: a systematic review and meta-analysis. PLoS One 2015;10:e0138066.

17. O'Brien KK, Tynan AM, Nixon SA, et al. Effectiveness of aerobic exercise for adults living with HIV: systematic review and metaanalysis using the Cochrane Collaboration protocol. BMC Infect Dis 2016;16:182

18. Fazeli PL, Marquine MJ, Dufour C, et al. Physical Activity is Associated with Better Neurocognitive and Everyday Functioning Among Older Adults with HIV Disease. AIDS Behav 2015;19:1470-7.

19. O'Brien KK, Solomon P, Trentham B, et al. Evidence-informed recommendations for rehabilitation with older adults living with HIV: a knowledge synthesis. BMJ Open 2014;4:e004692.
20. Vancampfort D, Mugisha J, De Hert M, et al. Sedentary Behavior in People Living With HIV: A Systematic Review and Meta-Analysis. J Phys Act Health 2017;14:571-7.

21. Vancampfort D, Mugisha J, De Hert M, et al. Global physical activity levels among people living with HIV: a systematic review and metaanalysis. Disabil Rehabil 2018;40:388-97.

22. Sallis JF, Owen N, Fisher E. Ecological models of health behavior. Health behavior: Theory, research, and practice 2015;5:43-64.

23. McLeroy KR, Bibeau D, Steckler A, et al. An ecological perspective on health promotion programs. Health Educ Q 1988;15:351-77.

24. Macera CA, Cavanaugh A, Bellettiere J. State of the Art Review: Physical Activity and Older Adults. Am J Lifestyle Med 2017;11:42-57.

25. Trost SG, Owen N, Bauman AE, et al. Correlates of adults' participation in physical activity: review and update. Med Sci Sports Exerc 2002;34:1996-2001.

26. Bauman AE, Reis RS, Sallis JF, et al. Correlates of physical activity: why are some people physically active and others not? Lancet 2012;380:258-71.

27. Schutzer KA, Graves BS. Barriers and motivations to exercise in older adults. Prev Med 2004;39:1056-61.

28. Simonik A, Vader K, Ellis D, et al. Are you ready? Exploring readiness to engage in exercise among people living with HIV and multimorbidity in Toronto, Canada: a qualitative study. BMJ Open 2016;6:e010029.

29. Ley C, Barrio MR, Leach L. Social-ecological, motivational and volitional factors for initiating and maintaining physical activity in the context of HIV. Open AIDS J 2015;9:96-103.

30. Webel AR, Perazzo JD, Dawson-Rose C, et al. A multinational qualitative investigation of the perspectives and drivers of exercise and dietary behaviors in people living with HIV. Appl Nurs Res 2017;37:13-18

31. Nguyen AL, Lake JE, Reid MC, et al. Attitudes towards exercise among substance using older adults living with HIV and chronic pain. AIDS Care 2017;29:1149-52.

32. Roos R, Myezwa H, van Aswegen H. "Not easy at all but I am trying": barriers and facilitators to physical activity in a South African cohort of people living with HIV participating in a home-based pedometer walking programme. AIDS Care 2015;27:235-9.

33. Montoya JL, Wing D, Knight A, et al. Development of an mHealth Intervention (iSTEP) to promote physical activity among people living with HIV. J Int Assoc Provid AIDS Care 2015;14:471-5.

34. Neff HA, Kellar-Guenther Y, Jankowski CM, et al. Turning disability into ability: barriers and facilitators to initiating and maintaining exercise among older men living with HIV. AIDS Care 2018:1-5.

35. Justice AC. HIV and aging: time for a new paradigm. Curr HIVIAIDS Rep 2010;7:69-76.

36. Brown D, Claffey A, Harding R. Evaluation of a physiotherapy-led group rehabilitation intervention for adults living with HIV: referrals, adherence and outcomes. AIDS Care 2016;28:1495-505.

37. Nosrat S, Whitworth JW, Ciccolo JT. Exercise and mental health of people living with HIV: A systematic review. Chronic IIIn 2017:13:299-319.

38. Vancampfort D, Mugisha J, Richards J, et al. Physical activity correlates in people living with HIV/AIDS: a systematic review of 45 studies. Disabil Rehabil 2018;40:1618-29.

39. Petróczi A, Hawkins K, Jones G, et al. HIV Patient Characteristics that Affect Adherence to Exercise Programmes: An Observational Study. Open AIDS J 2010;4:148-55

40. McAuley E, Szabo A, Gothe N, et al. Self-efficacy: implications for physical activity, function, and functional limitations in older adults. Am J Lifestyle Med 2011;5:361-9.

41. Koeneman MA, Verheijden MW, Chinapaw MJ, et al. Determinants of physical activity and exercise in healthy older adults: a systematic review. Int J Behav Nutr Phys Act 2011;8:142.

42. Gray L, Chalabaev A, Durant J, et al. Exercise stereotypes and fatigue in people living with HIV: does self-efficacy play a mediating or a moderating role? Arch Public Health 2018;76:23.

43. Schrimshaw EW, Siegel K. Perceived barriers to social support from family and friends among older adults with HIV/AIDS. $J$ Health Psychol 2003;8:738-52.

44. Lindsay Smith G, Banting L, Eime R, et al. The association between social support and physical activity in older adults: a systematic review. Int J Behav Nutr Phys Act 2017;14:56.

45. Prince SA, Reed JL, McFetridge C, et al. Correlates of sedentary behaviour in adults: a systematic review. Obes Rev 2017;18:915-35.

46. Thornton CM, Kerr J, Conway TL, et al. Physical Activity in Older Adults: an Ecological Approach. Ann Behav Med 2017;51:159-69. 